\title{
EXPLORANDO EL SIGNIFICADO CULTURAL DE LA NUEVA RURALIDAD EN CASTILLA Y LEÓN: UN CASO DE ESTUDIO INEXPLORADO
}

\author{
EXPLORING THE CULTURAL SIGNIFICANCE OF THE NEW RURALITY IN \\ CASTILLA AND LEÓN: AN UNEXPLORED CASE OF STUDY
}

\author{
Almudena Moreno Mínguez ${ }^{1}$
}

\begin{abstract}
Los resultados que se muestran, a continuación, son el resultado del trabajo de investigación realizado en una zona rural de montaña en la Comunidad de Castilla y León en España, concretamente en el "Guadarrama segoviano" desde un enfoque socioantropológico. La metodología de investigación aplicada en este estudio se ha basado en el análisis documental y la realización de entrevistas abiertas semiestructuradas a informantes estratégicos sobre las tradiciones e identidad cultural habiéndose realizado un total de 15 entrevistas. El objetivo fundamental de este artículo ha sido analizar la ambivalencia cultural de la "nueva ruralidad" desde una perspectiva sociológica y antropológica. La conclusión general es que los informantes entrevistados recuerdan con nostalgia un pasado cultural de tradiciones que está en vías de extinción, además de no identificarse con la denominada "nueva ruralidad", lo que puede interpretarse como un efecto ambivalente de la "hibridación cultural".
\end{abstract}

Palabras claves: tradiciones culturales, cambio cultural, modernización, nueva ruralidad.

The findings presented here are the result of a socio-anthropological research conducted in a rural mountain area in the Community of Castilla and León in Spain, specifically in the "Segovia Guadarrama". The research methodology applied in this analysis is based on document analysis and open-ended interviews with informants about traditions. A total of 15 interviews were conducted. My main objective was to analyse, from a sociological and anthropological perspective, the cultural ambiguity of the concept of "new rurality". The main conclusions are that the informants interviewed nostalgically remember a cultural past of traditions that are in danger of extinction, without identifying themselves with the so-called "new rurality". This can be interpreted as an ambiguous effect of "cultural hybridization".

Key words: Cultural traditions, cultural change, modernization, new rurality.

En los últimos años estamos asistiendo a un proceso de nuevas relaciones entre lo urbano y lo rural que están transformando los universos sociales y culturales, que han dejado de ser dicotómicos para convertirse en ambivalentes y en continua reconstrucción, adaptación y cambio. En las décadas de 1930 a 1940 se pasó de estudiar los pueblos lejanos y exóticos a estudiar ámbitos locales más cercanos geográficamente, dando lugar a la creación de una antropología del campesinado ibérico que poco a poco fue incorporando la metodología de una antropología histórica y una antropología relacional enfocada en la perspectiva local-global. De esta forma los antropólogos se han centrado fundamentalmente en España en el análisis del patrimonio cultural y natural y en menor medida en una antropología basada en el análisis de la comunidad local para el desarrollo rural. Este es precisamente uno de los objetivos que presentamos en este estudio.

Entre las primeras aportaciones de los estudios antropológicos sobre la ruralidad destacan los estudios sobre el campesinado realizados por Wolf (1975), y las contribuciones de Arensberg y Kimball (1988), cuyo foco de atención se centraba fundamentalmente en analizar la realidad del campesinado desde el eje Nación-Estado a partir de una mirada local y de proximidad cotidiana. Estos estudios situarán al campesinado -como sujeto de estudio- en el contexto de determinaciones nacionales e internacionales, lo que aproxima el foco de estudio a los efectos de la globalización en la ruralidad y vida cotidiana de los campesinos, así como su relación con el entorno social y natural más inmediato. De esta forma en la antropología se superó la idea de que lo local se interpreta a partir de lo local, para pasar a interpretar

1 Universidad de Valladolid, Departamento de Sociología y Trabajo Social, Campus María Zambrano, Plaza de la Universidad s/n Segovia, 40005, España. almudena@soc.uva.es 
lo local en la complejidad de las relaciones dinámicas y dialécticas con los procesos globales.

En el caso de la Península Ibérica los estudios campesinos realizados desde la antropología (Leal 2000; Narotzky 1991; entre otros), han contribuido por un lado a avanzar en los estudios sobre la diversidad cultural dentro de un mismo territorio y por otro lado a la superación de metodologías unidimensionales para interpretar los contextos rurales dentro de las tradiciones históricas, introduciendo nuevas perspectivas que relacionan las transformaciones rural-urbano en relación con los grandes procesos globalizadores desde una perspectiva sistémica. También han contribuido a ir más allá de la dicotomía rural-urbano para analizar el continuo rural-urbano de una forma dinámica, interrelacionada y compleja destacando la transformación y en su caso la pérdida de las tradiciones culturales asociadas a un modo de vida en rápida y continua transformación desde los años sesenta en España.

Los estudios sobre la ruralidad en España se han realizado fundamentalmente a partir de análisis etnográficos que difícilmente han podido captar las interrelaciones entre los procesos globalizadores y las dinámicas transformadoras de las relaciones culturales, puesto que utilizan una metodología descriptiva y en parte estática. Es pertinente en este punto introducir la reflexión de Geertz (1991) para quien los antropólogos no estudian aldeas, sino que estudian en las aldeas, diferenciando entre el objeto y el ámbito local de estudio. En la misma línea se sitúa la crítica de Narotzky (2001) al analizar los estudios de comunidades rurales realizados en territorio español y concluir que estos se asientan en continuas polaridades y obvian los contextos de transformación.

En los estudios antropológicos sobre las relaciones rural/urbano realizados en el Estado español, nos encontramos con obras clásicas sobre esta problemática específica (Caro Baroja 1963, 1966; Kenny 1962), otras más recientes que se centran en los vínculos urbano/rurales desde el punto de vista de la ciudad (Cátedra 2008; Rodríguez Campos 2008), otros que examinan procesos de cambio (Bretón et al. 1997; Pereiro 2005) o también procesos de reducción de los servicios escolares en el ámbito rural (Prado 2007). Por tanto, cabe señalar que aunque el campesinado sigue siendo un objeto de estudio fundamental en la antropología para la compresión de la nueva ruralidad, este colectivo se ha transformado en nuevos perfiles y agentes sociales como los "neorrurales", "retornados", "población flotante", etc., y como consecuencia de ello muchos de los sistemas culturales que conformaban las estructuras sociales tradicionales han pasado a ser residuales. Esto exige un cambio en las formas de interpretación de dichas realidades tal y como han expresado Roseman et al. (2011):

...cuando tratamos de acercarnos a las 'nuevas ruralidades' tenemos que pensar en cómo los espacios rurales, sus habitantes y los que atraviesan estos espacios con más o menos duración están todos situados dentro de un sistema global en términos de economía política, ecología, redes sociales, y significaciones culturales (Roseman et al. 2011:2166).

Por su parte, Ruiz y Delgado (2008) cuestionan el potencial explicativo de la antropología clásica para entender las transformaciones que están aconteciendo en la nueva ruralidad. Desde nuestro punto de vista, y en concordancia con autores como Roseman et al. (2011), el estudio de los nuevos procesos culturales, sociales y económicos que están aconteciendo en los ámbitos rurales requieren el análisis interpretativo de los flujos urbano-rurales, sus resistencias y adaptaciones culturales a los contextos de globalización.

En este contexto de interpretación sobre las consecuencias del cambio socioeconómico emerge lo que se denomina el proceso de "hibridación cultural" como nueva línea de investigación. Por tal, los investigadores se refieren a la dinámica de cambio cultural que hunde sus raíces en las profundas transformaciones experimentadas en los ámbitos rurales como consecuencia de los procesos modernizadores e industrializadores iniciados a finales del siglo XIX. En concreto, García Canclini entiende por hibridación:

los procesos socioculturales en los que estructuras o prácticas discretas, que existían en forma separada, se combinan para generar nuevas estructuras, objetos y prácticas (García Canclini 2004:3).

Estos procesos conducen en muchos casos a relativizar la noción de identidad local. Son escasos los estudios sobre costumbres populares y tradiciones 
realizados sobre la base del concepto de hibridación, puesto que la literatura antropológica ha resaltado el hecho de que la identidad cultural es un objeto de estudio en sí mismo homogéneo, con unos contornos definidos y derivados de las tradiciones construidas social y culturalmente a partir de la relación que los individuos mantienen con el entorno natural para sobrevivir. Sin embargo, algunos investigadores proponen, por eso, desplazar el objeto de estudio "de la identidad a la heterogeneidad y la hibridación intercultural" (Goldberg 1994:6). La formas en las que los miembros de cada grupo se apropian de los repertorios heterogéneos de bienes, discursos y costumbres en los circuitos de producción genera nuevas formas de vida asentadas sobre tradiciones culturales del pasado que se transforman y reinventan para adaptarse a los nuevos tiempos. El concepto de hibridación cultural resulta útil en las investigaciones antropológicas para abarcar conjuntamente los procesos de transformación intercultural de mezclas entre lo artesanal y lo industrial, lo culto y las tradiciones. Desde esta perspectiva cabe interpretar las transformaciones acontecidas en los entornos rurales como el resultado de construcciones híbridas entre manifestaciones culturales de los actores modernos y las tradiciones del pasado en el contexto más amplio de globalización y modernización (García Canclini 2004; García Canclini y Roncagliolo 1989). En tales procesos ambivalentes y contradictorios resultantes de la hibridación cultural permanecen prácticas culturales y sociales como las "tradiciones populares" asociadas a la fiesta, los rituales religiosos, familiares o productivos, donde los procesos de hibridación mezclan lo tradicional con lo moderno, introduciendo las tradiciones en circuitos culturales y turísticos diversos en los que se aprovechan las tradicionales manifestaciones culturales resultantes de esos procesos de hibridación cultural como reclamos turísticos.

El concepto de hibridación también se ha utilizado desde la geografía para explicar las nuevas combinaciones surgidas de las imbricaciones tecnológicas, demográficas y naturales que se ciernen sobre el ámbito rural, donde las fronteras entre los procesos y fenómenos cada vez son más complejas y difusas (Ribas y Sauri 2006:290). Si bien el complejo concepto de "hibridación cultural" resulta limitado para explicar la trasformaciones económicas, territoriales, naturales y culturales del ámbito rural en Castilla y León, sí al menos resulta útil para acercarnos a la comprensión interpretativa de cómo los sujetos y actores protagonistas de la transformación rural describen el cambio en términos de dualidad, contradicción, nostalgia y expectativas futuras; manifestaciones propias de la hibridación cultural que surgen de la combinación de viejas y nuevas identidades locales.

El interés que tiene este concepto para nuestro análisis está en el hecho de que las prácticas culturales asociadas a las tradiciones se encuentran asociadas a las formas cómo estas se construyen (generalmente a través de un proceso de hibridación cultural), asî como a las vinculaciones que mantienen con ciertos patrones de identidad local. Este planteamiento es clave para entender cómo las tradiciones culturales no responden únicamente a patrones normativos establecidos previamente, sino también a procesos complejos de hibridación cultural.

Muchos de los estudios antropológicos que se hicieron sobre el medio rural en España partían del paradigma teórico de la "identidad" para explicar las relaciones entre lo urbano y lo rural como resultado del proceso modernizador e industrializador. Sin embargo no contamos con estudios específicos desde la antropología que hayan estudiado este proceso de hibridación cultural en Castilla y León, más allá de los estudios realizados por los folcloristas sobre las costumbres y modos de vida en Castilla y León (Díaz 1984, 1988; Velasco 2001). Por tanto, este trabajo trata de ir más allá de los trabajos etnográficos realizados en Castilla y León sobre la definición y transformación de lo rural focalizados básicamente en una perspectiva sectorial de lo rural. Partiendo de la revisión crítica de las formulaciones teóricas sociológicas y antropológicas sobre la "nueva ruralidad" y el fenómeno de la "hibridación cultural", los aportes aquí presentados tratan de visibilizar los nuevos modelos de desarrollo introduciendo la dimensión territorial y cultural a través de la perspectiva de los sujetos en el contexto de los complejos procesos de transformación cultural a nivel local que está produciendo la globalización en el territorio objeto de estudio.

\section{Los Componentes Territoriales del "Guadarrama Segoviano": La Dualidad del Modelo Territorial}

Para comprender adecuadamente las transformaciones que se han producido en el ámbito rural estudiado hay que situar las características territoriales del área objeto de estudio. La sierra del 
Guadarrama forma parte de la provincia de Segovia, situada en Castilla y León. El territorio objeto de estudio está formado por 42 municipios a lo largo de la Sierra. El "Guadarrama segoviano" constituye un espacio caracterizado por un importante proceso de regresión demográfica durante la segunda mitad de la anterior centuria, no superando ninguno de los municipios los 1.000 habitantes.

El territorio objeto de esta investigación sobre la nueva ruralidad enfocado en un estudio de caso en la región de Castilla y León se inscribe desde el punto de vista de su integración en el espacio físico de la provincia de Segovia, en la vertiente septentrional -o segoviana- del Guadarrama. Esta es, por tanto, su enclave geográfico de referencia; dando por buena esta nomenclatura para definir a todo el conjunto serrano como la "Sierra de Segovia"1. En esencia, es en esta parte de "Sierra" en la que mejor se conservan los vestigios, las herencias y las huellas de la cultura y la sociedad serrana tradicional ${ }^{2}$. En este territorio se ha forjado la construcción histórica de una comunidad relacional, hoy, singularizada. De ahí emerge la elección de esta parte de la "Sierra", como ámbito específico a estudiar, tanto en su dimensión territorial como cultural.

El área de la "Sierra" objeto de atención en este estudio se inscribe por tanto en el espacio físico comprendido por los piedemontes de Pedraza y Segovia y el Guadarrama oriental -los Montes Carpetanos-. Dentro de un territorio que, desde el punto de vista funcional, ha sido definido como "regresivo" en términos de comportamiento demográfico y dinámica socioeconómica, con el sector agrario en crisis y una cierta readaptación en forma de "presencia creciente" de nuevas actividades, en particular las de índole turística y residencial, como nuevas formas de identidad cultural que conviven con las tradicionales en vías de extinción. Es el signo del agotamiento socioeconómico y demográfico de los tiempos cambiantes en municipios como Casla, Prádena, Arcones, Matabuena, Gallegos, Aldealengua de Pedraza, Navafría, Torre Val de San Pedro, Santiuste de Pedraza, Collado Hermoso, Sotosalbos, Santo Domingo de Pirón y Basardilla (Figura 1).

Por tanto, el área objeto de estudio en esta investigación se sitúa al este de la capital segoviana, en el espacio serrano bajo la dominancia comarcal de Riaza y de los municipios de Santo Tomé del Puerto, Cerezo de Arriba o Cerezo de Abajo, en una suerte de zona fuertemente ruralizada y débilmente poblada
(Figura 2). Se define como un área "regresiva con los sectores tradicionales en crisis y una presencia creciente de actividades transformadoras" (Díez Mayoral 2003; Troitiño 1990). Con todo, se trata de un territorio definido en su momento como de "fuerte dinámica de cambio" provocada por la irrupción masiva de nuevas funciones -la turística y la residencial-por encima del resto de actividades (Plaza et al. 2010). Por tanto, la dicotomía territorial y sectorial es la característica determinante en el "Guadarrama segoviano".

Desde el punto de vista administrativo este territorio objeto de estudio es un espacio fragmentado en pequeños municipios dotados de un nivel de equipamientos y de servicios básicos muy elemental, muchos de ellos apenas cuentan con el consultorio médico rural, que no atiende todos los días, y carecen de cualquier tipo de establecimiento de alimentación. Es un territorio que históricamente fue articulado por "villas" ajenas o pertenecientes al ámbito serrano como Sepúlveda o Turégano, entre las primeras; Pedraza y Prádena entre las segundas. De hecho ninguno de los municipios es siquiera considerado como centro de servicios de tercer nivel (el más bajo de todos) en las diferentes clasificaciones y tipologías municipales establecidas a tal efecto

\section{El proceso de aculturación en el "Guadarrama segoviano": un estudio de caso}

Desde los años ochenta la transformación de la estructura productiva como consecuencia de la denominada "revolución industrial" (Nadal 1975) afectó de lleno a las tendencias demográficas y poblacionales de la ruralidad castellano y leonesa y concretamente a la provincia de Segovia y por tanto a las tradiciones y prácticas culturales. Son numerosos los estudios antropológicos y etnográficos que dan cuenta de las transformaciones experimentadas por el medio rural en Castilla y León, tanto en la estructura productiva como en el sistema de estratificación y las formas de vida cultural ligados a tales formas de estructuración social, tal y como han documentado numerosos estudios para Castilla y León (Velasco Maillo 1998, 2001, 2004). Un ejemplo de este tipo de análisis etnográficos es el magistral análisis realizado por Aceves (1973) sobre el pueblo de El Pinar en Segovia. En este libro se relatan los complejos procesos de transformación social, económica y cultural a los que tuvieron que 


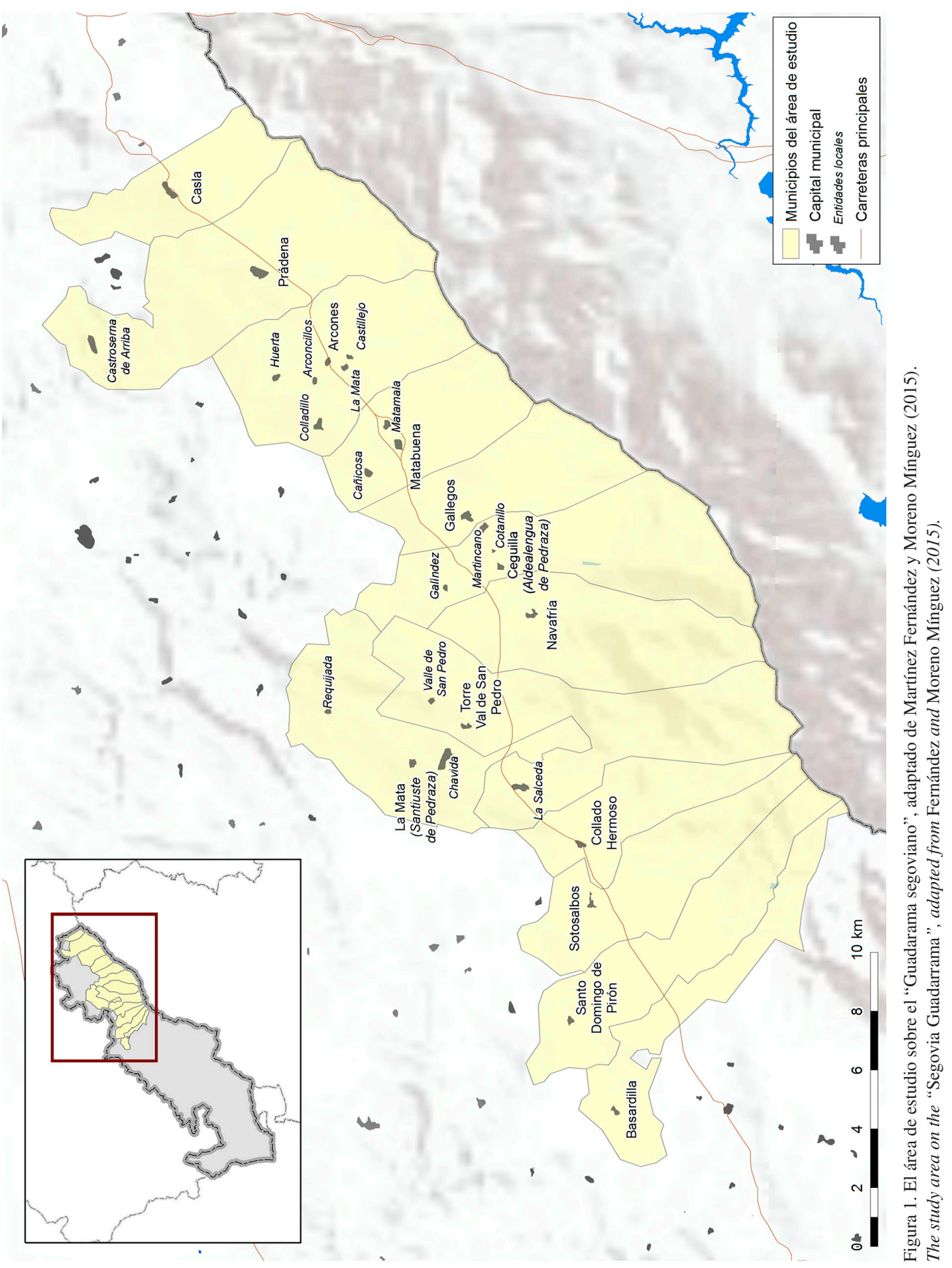




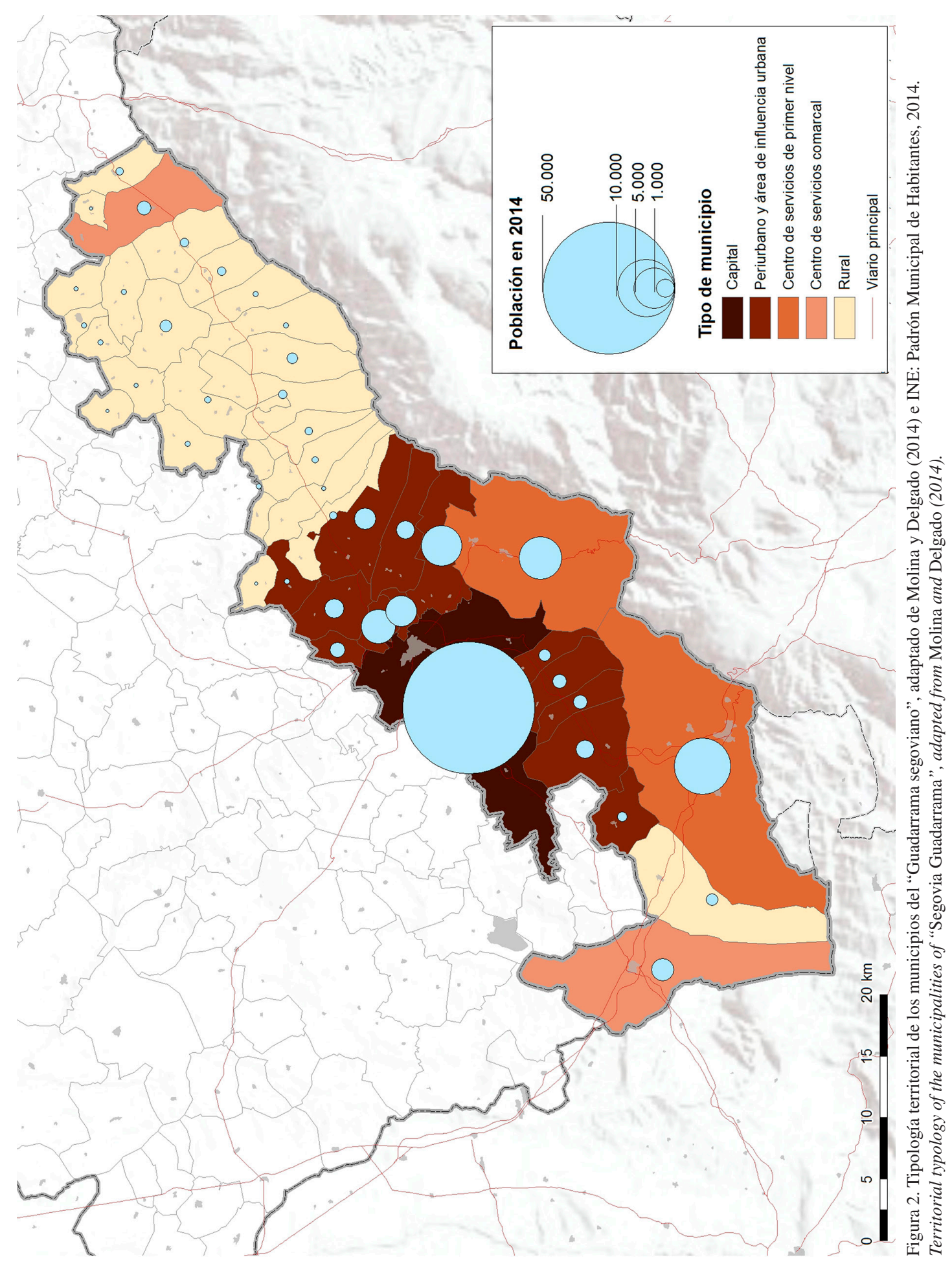


hacer frente los habitantes de un pueblo en pleno proceso de industrialización y cambio social.

Esto supuso lo que algunos investigadores han denominado "un giro cultural" en las tradiciones y costumbres de las ámbitos rurales como consecuencia de la emigración hacia las ciudades y la desagrarización. Tal y como muy bien anotó Geertz (1991):

El hombre es un animal inserto entre tramas de significados que él mismo ha tejido; considero que la cultura es esa urdimbre y que el análisis de la cultura ha de ser, por lo tanto, no una ciencia experimental en busca de leyes, sino una ciencia interpretativa en busca de significados (Geertz 1991:20).

Esta afirmación del antropólogo Clifford Geertz se convirtió en el punto de partida de la nueva antropología en lo que se denominó el "giro cultural" de las ciencias sociales (Bonnel y Hunt 1999). Esta nueva perspectiva metodológica en antropología desplazó el interés en estudiar la estructura social hacia el estudio de cuestiones relacionadas con los símbolos, los rituales, los discursos y prácticas culturales. Desde esta perspectiva la cultura se considerará como algo representacional y textual (Sewell 1992). En función de estos antecedentes teóricos y metodológicos, los trabajos antropológicos de los últimos veinte años coinciden en una definición común de "cultura", entendida como un conjunto de códigos o repertorios públicos comunes que intervienen en la capacidad de las personas para pensar, interpretar y comunicar ideas relacionadas con el entorno en el que viven (Eliasoph 1988; Sewell 1992; Somers y Block 2005; Swilder 1986). Desde esta perspectiva la cultura responde a sistemas simbólicos compartidos por ciertos grupos que son además prácticas sociales (Fernández Mosteyrin y Morán 2014). Esta interpretación antropológica de la realidad cultural dio lugar a numerosos dilemas teóricos sobre las relaciones entre estructura y cultura (Archer 1988) y a una nueva evaluación de los indicadores existentes para interpretar los significados de las culturas y las tradiciones (Biernacki 2012). Esto fundamentalmente supuso poner en el centro del debate antropológico el análisis de las dimensiones culturales de la praxis social, lo que llevó poner en cuestión la aceptación de la cultura y las tradiciones como un conjunto de códigos lingüísticos compartidos que reducían la interpretación de las culturas y las tradiciones a una mera "lectura de signos". A partir de este momento se empieza a considerar que las culturas y tradiciones son formas históricas creadas y reproducidas a partir de la práctica social (Battani et al. 1997).

En consecuencia con este nuevo repertorio teórico y metodológico desarrollado en antropología se empezaron a realizar estudios empíricos que fueron más allá de la mera interpretación de los códigos de significados básicos, poniendo el foco de atención en cómo los actores sociales interactúan entre sí y con el entorno para satisfacer sus necesidades de subsistencia, socialización y comunicación a partir de múltiples significados. Esta nueva forma de entender lo cultural se fundamenta en el análisis de la diversidad de formas culturales a través de las cuales los individuos y grupos recurren a diversos artificios para sobrevivir al día a día, creando, interpretando y reinventando significados. En definitiva este nuevo giro interpretativo se fundamenta en lo que se ha denominado "la cultura de la acción", que dio lugar a numerosas investigaciones empíricas en las que los discursos de los actores sociales creadores de significados y tradiciones constituyen una pieza clave para el análisis (Fernández Mosteyrin y Morán 2014). Por tanto, la piedra angular para estos análisis culturales es el discurso narrativo producido por la interacción de los actores a través de la actividad humana cotidiana y que dota de significado a la realidad que nos rodea (Maines 2000; Polletta 2008).

En definitiva, esta comarca del "Guadarrama segoviano" bien podría ser considerada con algún matiz como una "zona de refugio" en la que se han gestado tradiciones culturales históricas a partir de la reproducción de la propia práctica social e interacción social. Esta noción se refiere a una zona que ha mantenido tradicionalmente una economía vinculada a la tierra o la ganadería que no ha tenido vías de comunicación cultural con entornos culturales significativamente distintos $\mathrm{y}$, por lo tanto, no ha experimentando grandes transformaciones externas (Aguirre Beltrán 1967). La consecuencia más inmediata del proceso modernizador y globalizador sobre esta "zona refugio" ha sido el despoblamiento y el abandono de muchas tradiciones que aún así permanecen en el imaginario social y colectivo pero que no se han podido recuperar. En estas zonas apenas se mantienen en vigor aspectos sociales y culturales tradicionales. Sin embargo, de acuerdo 
con Montes Pérez (2001), el elemento distintivo de interés en estas zonas es el proceso acelerado de transformación y de cambio sociocultural que han experimentado, dando lugar a nuevas formas de relación social, cultural, simbólica y económica que aún están por reconstituirse y visibilizarse como una forma de economía cultural vinculada a este territorio. Este proceso que se observa en las regiones de refugio las convierte en objetos de estudio especialmente interesantes para el análisis antropológico, puesto que evidencian las bases procesuales y contextuales sobre las cuales se construyen los mecanismos de respuesta en una sociedad cada vez más globalizada, donde la cultura y las tradiciones tienen un papel relevante para explicar el cambio social y cultural acontecido en estas zonas rurales.

En base a estas consideraciones previas, nuestro objetivo es buscar la "cultura en acción" de esta "zona refugio" de la "Sierra", a través de las dinámicas culturales que está produciendo el proceso de hibridación cultural y que sus actores protagonistas identifican a través de la recuperación de sus discursos. Nos referimos con ello al problema básico de convertir un planteamiento teórico en una estrategia de investigación antropológica. Partimos de la idea de que la cultura es una práctica, por lo que la "cultura en acción" debe aprehenderse en procesos sociales que ocurren en contextos sociales específicos. En nuestro caso hemos seleccionado un espacio singular como es la porción oriental del "Guadarrama segoviano" para analizar cómo los individuos y los grupos han interactuado con el entorno dando lugar a formas de expresión cultural diversas previas al proceso industrializador; dinámicas que pretendemos identificar en un contexto de hibridación cultural y de cambio acelerado que transformó drásticamente el significado de la ruralidad y de las tradiciones que durante años habían dado sentido a la vida de estos individuos.

\section{Nota Metodológica}

La información obtenida para documentar el estudio fue obtenida por revisión de documentos históricos y 15 entrevistas semiestructuradas ${ }^{3}$, cuyo guión se elaboró a partir de las conclusiones obtenidas en la observación participante realizada previamente en el ámbito territorial objeto de estudio durante nueve meses. Los ejes temáticos que orientaron las entrevistas se basaron en el corpus teórico elaborado a partir de los documentos históricos consultados y la propia observación participante y fueron los siguientes: actividades productivas, manifestaciones culturales, relaciones familiares, relaciones con la comunidad y prácticas de ocio. Estas entrevistas fueron realizadas a informantes estratégicos de las diferentes localidades de la "Sierra" durante el invierno y el verano de 2015 aplicando el principio de saturación de la información. El análisis del discurso se sistematizó a partir de las categorías previamente definidas con el fin de reconstruir parcialmente la historia oral del cambio en este territorio desde la etapa previa a la industrialización (años cincuenta) y la masiva emigración del campo a la ciudad (años sesenta-setenta) hasta el momento de realización de las entrevistas, completando este análisis con la información complementaria obtenida en las fuentes secundarias consultadas. Nos interesaba que los participantes explicaran su experiencia personal, sus impresiones y valoraciones sobre el tema de estudio. La lógica del guión utilizado para realizar las entrevistas tenía como fin epistemológico focalizar el eje central de la investigación en la transformación de lo rural desde la vivencia personal de los actores protagonistas del cambio en el territorio objeto de estudio.

\section{La nueva ruralidad vista por lo agentes sociales: el ambivalente significado del cambio social y cultural}

En el pasado, el concepto de desarrollo se vinculaba a la noción de progreso y transformaciones acontecidas en el territorio que iban desde la transformación en el sector agrícola-ganadero, de lo tradicional a lo moderno, de lo atrasado a lo próspero, en definitiva desde lo rural hacia lo urbano. Si se examinan los principales temas abordados por los autores clásicos de la sociología rural, no es posible encontrar un enfoque específico del tema. Más bien los investigadores han optado por analizar las consecuencias que tiene la industrialización y la urbanización en la sociedad rural en todos los ámbitos asociados a esta nueva situación, más que focalizar su atención en los efectos que esta transformación está teniendo en los estilos de vida, actitudes y valores de los residentes en un ámbito rural en continua y profunda transformación (Töennies 1973; Sorokin et al. 1981 [1930]). En esta indefinición teórica aparece el concepto de "nueva ruralidad" debido a que se tiende a confundir la definición de procesos 
con la definición de conceptos. De hecho en la literatura encontramos recurrentemente conceptos como desarrollo territorial, local, sostenible y rural. Si bien estos se refieren a los procesos de transformaciones productivas e institucionales experimentados por estos territorios para adaptar sus economías a mercados dinámicos y favorecer así el asentamiento de población, sin embargo estos conceptos no contemplan las dinámicas vivenciales experimentadas por los residentes en este proceso de transformación del mundo rural. Nuestro punto de partida en este artículo ha sido considerar lo rural como un tipo de relación social específica que predomina en territorios con una baja densidad de población relativa debido a los procesos de transformación socioterritorial ocurridos durante la industrialización en España y así poder acercarnos a la comprensión de las dinámicas sociales que han vivido los agentes sociales del "Guadarrama segoviano" implicados en ese proceso de cambio social.

De acuerdo con Kay (2009), lo "nuevo" y lo "viejo" se siguen confundiendo muchas veces de manera recurrente sin que los sociólogos y antropólogos de la nueva ruralidad hayan logrado descifrar adecuadamente el significado de esta nueva situación para los habitantes que residen en estos territorios rurales. Esta expresión refleja el escepticismo de algunos investigadores sobre el concepto de la nueva ruralidad. De acuerdo con Gómez (2002:12) se han producido múltiples transformaciones relevantes en el sector rural llegando a la conclusión final de que "quizás, lo nuevo es que ahora se mira una realidad que antes se ignoraba".

Otro aspecto que la nueva ruralidad puso de manifiesto fue la desaparición de la diferencia entre el mundo rural y el urbano. El concepto tradicional basado en la división rural-urbano ha sido cuestionado debido a la creciente interacción de los dos campos de acción e interacción. En el pasado algunos autores hablaron de "ciudades de campesinos" o "el campo en la ciudad" debido al aumento de la migración del campo a las ciudades (Linck 2001; Roberts 1978). Actualmente, sin embargo, la situación es más plural y no solo los agricultores y ganaderos se trasladan a las ciudades sino que también los habitantes de las ciudades migran al campo, y con esto surgen nuevos asentamientos urbanos en el campo formando lo que se ha dado en llamar "rurbanización" (Delgado 1999) o asentamientos "neorrurales"
(Nogué 2005). Con la proliferación en las últimas décadas de pequeñas comunidades urbanas y de ciudades intermedias, la interrelación con las áreas agrícolas circundantes ha aumentado (Schejtman 1999). Este fenómeno ha sido muy poco estudiado en la provincia de Segovia y concretamente en el ámbito serrano que nos ocupa en este estudio.

Las diferentes concepciones normativas y formales sobre la "nueva ruralidad" aquí presentadas no han sido suficientemente aplicadas para explicar la multifuncionalidad que caracteriza a los nuevos procesos de transformación en el mundo rural en Castilla y León, así como la forma de entender el territorio y la cultura. Por lo tanto este análisis trata de aplicar las nociones relativas a la "nueva ruralidad" e "hibridación cultural" a un ámbito territorial concreto para interpretar cómo los sujetos en sus redes de interacción social entienden el territorio y las transformaciones experimentadas en el ámbito rural local a partir de la articulación con los procesos globales en los que se manifiestan e identifican los desajustes económicos, territoriales y culturales propios del proceso globalizador (Chiriboga 2001; Gómez Echenique 2002)4.

\section{Identidad y cambio}

En relación con las particularidades del proceso de hibridación cultural presentadas en este artículo, la definición de lo rural no se puede entender sin relacionarlo con lo urbano y con el proceso de transformación experimentado por las comunidades rurales y así lo expresan nuestros entrevistados. En general, los discursos de los entrevistados evidencian que la nueva ruralidad no se puede entender ni gestionar adecuadamente sin entender la vieja ruralidad. La nueva ruralidad se está construyendo bajo las ruinas de los viejos cimientos de la vieja ruralidad como respuesta a la crisis de identidad y del limitado bienestar que generan las grandes urbes. Los entrevistados son conscientes de que este proceso de recuperación no se puede hacer sin remitirnos a la articulación entre el intercambio de bienes y servicios del mundo rural con el mundo urbano. Por lo tanto, los vestigios y formas de vida del pasado se han convertido en los andamiajes claves para sustentar un nuevo modelo de ruralidad en continuo diálogo e intercambio con el ámbito urbano.

Los entrevistados han señalado diversos iconos de la cultura local que remiten a formas tradicionales 
de subsistencia y que forman parte de la identidad colectiva y cultural de unos espacios que hoy están abocados a una transformación inminente que se debate entre el olvido y la recuperación de formas tradicionales de vida como legado cultural material e inmaterial para convertirse en atractivos turísticos o espacios alternativos de vida.

Las ocupaciones productivas son el hilo vertebrador de las formas de vida en la "Sierra", en un esfuerzo titánico por intentar sacar el máximo partido al entorno para sobrevivir y vivir. La mayoría de los entrevistados se refieren a los oficios de la zona vinculados fundamentalmente a la ganadería como articuladores de los estilos de vida. Uno de los más destacados es el referido a la oveja, la lana y la trashumancia hasta su decadencia en los años setenta. Este modo de vida vinculado a la oveja merina y presente en la mayoría de los pueblos serranos articulaba la vida familiar y la economía de las familias. Tal y como han expresado la mayoría de los entrevistados, los hombres se iban en el invierno a la Extremadura y las mujeres quedaban al cuidado de los hijos y de la economía familiar, por lo que la mujer tenía un protagonismo destacado en el trabajo en el campo y en el trabajo dentro del hogar. Era una forma de vida que hoy se ha perdido y que pone en valor el trabajo de las mujeres en el campo. La industrialización trajo consigo en España una nueva forma de familia en las ciudades en las que el hombre trabajaba en la industria y la mujer se ocupaba del cuidado de los hijos. Sin embargo, estos testimonios sobre la trashumancia evidencian que la mujer ha trabajado siempre en el campo, al menos en esta zona de la provincia de Segovia, por lo que el fenómeno de la incorporación de la mujer al mercado laboral no es algo nuevo.

De las entrevistas realizadas se deduce que la modernidad se funde con la tradición perdiéndose en muchos casos el origen y sentido de la tradición que se trata de recordar y recuperar por medio de la fiestas, los museos improvisados o algunas representaciones teatrales sobre los oficios del pasado, tal y como sucede con la representación teatral de la "siega" o la "matanza del cerdo", que congrega a los vecinos de estos pueblos. Tal y como ha señalado Alonso Ponga (1982), y como hemos comprobado en nuestras entrevistas, algunas de las tradiciones populares que aún se conservan tienen un ritual en parte ilógico y falto de sentido, puesto que han perdido parte de su simbolismo asociado a las formas de vida de los habitantes de estos pueblos, quedando reducidas a una mera costumbre que se transmite de generación en generación. Esto ocurre con la mayoría de las fiestas religiosas y paganas que se siguen celebrando en estos territorios y que no se recuerda su simbolismo (Caro Baroja 1963, 1967; Montesino González 1982). Estos rituales tradicionales contribuían a reproducir estrategias, diferenciales y diferenciadoras de jerarquización social, de sociabilidad e identidad sexual, grupal comunitaria o supracomunitaria, poniendo de manifiesto sus mecanismos de construcción social del espacio, de los géneros, de las edades, del sistema productivo y del universo simbólico-ideacional de las gentes que conformaban los modelos comunitaristas de la sociedad tradicional.

Si bien las fiestas y los tiempos marcados por la economía local de esta zona, basada fundamentalmente en la ganadería y la agricultura, marcaban tanto el ciclo vital y cotidiano de sus habitantes como su identidad (Pitt-Rivers 1961, 1984), también es cierto que esta adaptación estratégica al entorno también marcó un carácter y una identidad propia de estas gentes. Esta forja de la identidad ha sido muy bien descrita por Kavanagh (1986) para un pueblo castellano:

....la cuestión de la identidad es algo que aparece matizado por el simbolismo del espacio, con un constante intercambio entre la visión del mundo exterior y el mundo interior. Este simbolismo identitario aparece reflejado en las expresiones de los informantes entrevistados cuando se refieren a que "las gentes de los pueblos son cerrados y les cuesta mucho cambiar" (Kavanagh 1986:41).

Esa expresión denota cierto mimetismo de sus habitantes con el entorno que ha conformado un carácter y una identidad ligados a la ambivalencia de lo propio y lo extraño. Sin embargo, no debemos caer en el error de algunos antropólogos $\mathrm{y}$ folkloristas de referirnos a la sociedad tradicional como si fuera estática o inmóvil, describiendo el cambio social como si hubiera sido un fenómeno exclusivo causado por el despegue industrial y la modernización (Arnhold 1986). Nuestros informantes en cierta manera han respaldado esta idea con la ilusión de recordar con nostalgia la vida tradicional de siempre en continuidad con un pasado remoto y en armonía con el entorno (Díaz Viana 1984). 
Sin embargo, como han demostrado numerosos antropólogos, es necesaria cierta desmitificación de este hecho para documentar cómo los cambios que se han producido en las condiciones de vida han influido también en el cambio de las normas de comportamiento que se han mezclado con las nuevas, dando lugar a un modelo híbrido cultural que ha supuesto importantes progresos en el ámbito rural y societal de los pueblos de Castilla y León (Caro Baroja 1967; De Castro 1985). Por otro lado, investigadores como Díaz Viana han argumentado la falta de identidad de los castellanos y leoneses como resultado de un proceso de reificación permanente de lo que ha sido la vida en el pueblo sin plantearse reclamar una identidad territorial. De acuerdo con Díaz Viana (2010):

\section{...se reivindicaría volver a ser lo que -supuestamente- siempre se fue como pueblo, aunque nunca esa aspiración de identidad hubiera llegado a tener traducción territorial clara ni comprobación documental (Díaz Viana 2010:47).}

Este hecho simbólico ha marcado el devenir histórico de estos pueblos castellanos que después de la industrialización han visto reducir su población y la pérdida de sus formas tradicionales de vida. Nuestros informantes hacen una reclamación expresa de la pérdida de identidad de estos pueblos como conjunto de tradiciones pero no como una demanda territorial de reconocimiento de una entidad propia.

En base a estas interpretaciones narrativas, los pueblos de Castilla y León, y más concretamente los que nos ocupan en este estudio, se presentan en la primera década del siglo XXI como un territorio cuestionado y un pueblo que parece desentendido o ignorante de sí mismo (Díaz Viana 2010). Esto parece coincidir con la interpretación del sociólogo De Miguel, para quien los castellanos, al igual que los judíos, son un "pueblo-linaje". Esta comparación se debe a que unos y otros han tenido en común la "diáspora": "La población de los castellanos está constituida por el pueblo de Castilla y León y por los castellanos que residen fuera" (De Miguel y Moral 1984:37). Quizás esta sea la clave para entender cómo la permanencia singular de lo que aún pervive de las tradiciones de los pueblos de Castilla, y concretamente de los pueblos del "Guadarrama segoviano", no se puede entender sin las rememoraciones que hacen sus habitantes de las tradiciones pasadas pero desde otros territorios, ya sea desde la ciudad o desde otras regiones. Las tradiciones se han mantenido como recuerdos y reminiscencias en la diáspora de sus habitantes, de ahí la importancia que tienen estos trabajos de investigación para intentar aportar datos interpretativos sobre el significado de la transformación de lo rural, desde la perspectiva de los sujetos, en un territorio prácticamente inexplorado para la etnografía y la antropología, en un espacio cuya identidad colectiva no se basa únicamente en la pertenencia al territorio, sino en la identidad compartida de las tradiciones que permanecen en el imaginario social colectivo de unos habitantes que están en la "diáspora" y reviven las tradiciones desde la distancia o en estancias temporales vinculadas al ocio (De Miguel y Moral 1984).

\section{Tradición y modernidad}

Como se ha documentado por numerosos antropólogos y como han puesto de manifiesto los informantes entrevistados, la vida tradicional de estos pueblos serranos era muy vulnerable y precaria debido a los avatares de una economía de subsistencia y a las propias contradicciones (Arnhold 1986; Arnhold y Anthony 1981). Antes del desarrollismo industrial estos pueblos eran autosuficientes y sus vecinos tenían relativa sensación de continuidad con el pasado. La identidad del individuo estaba marcada por su ocupación productiva que venía determinada por la herencia. Por otra parte, los entrevistados han destacado que el "familismo" era la base fundamental de la organización social, junto con el paternalismo. Esto quiere decir que la división sexual del trabajo estaba claramente delimitada. Las mujeres se dedicaban a criar, cuidar a lo hijos y al trabajo del campo, mientras que los hombres de esta zona se ocupaban del ganado y, concretamente, eran los que hacían la "trashumancia" (Aitken 1947). Eran las propias condiciones de vida las que imponían estos roles de género. Si bien es cierto que las mujeres en la sociedad agrícola de Castilla y León de los años sesenta y setenta estaban más formadas que la media española, fundamentalmente a través de la vía del Magisterio, con el objetivo de buscarles una salida profesional más allá del campo, donde los hombres tenían reservada una posición (De Miguel 1984 ).

La familia era la principal forma de organización económica y social de estas sociedades agroganaderas. 
En torno a la familia giraba un imaginario social colectivo que impregnaba la vida de estos individuos. Quizás la forma cultural más conocida derivada de la organización familiar sea lo que se ha denominado "familismo". El familismo es una expresión cultural que se sustenta en estrategias familiares de supervivencia relacional basadas en la protección de los miembros de la familia, aunque estas estrategias familiares resulten disfuncionales para el bien público, lo que ha derivado en las sociedades del sur de Europa en diferentes modalidades de "clientelismo social, económico y político" (Cazorla 1994; Günes Ayata 1994). El concepto de "familismo amoral" como forma de organización social y económica tiene su referente en el trabajo de Banfield sobre una comunidad rural del sur de Italia en los años 40. En el caso de la provincia de Segovia, Aceves (1973) ha documentado magistralmente cómo el "familismo amoral" es un constructo cultural que ha regido la vida cotidiana de muchos de los habitantes de estos pueblos que tratan de hacer frente a las dificultades del día a día protegiendo a su entorno familiar, aunque esta forma de solidaridad familiar sea disfuncional para la comunidad y para el propio funcionamiento de las instituciones locales basadas en tradiciones que resultan anacrónicas al cambio social y que han sido incapaces de adaptarse a los nuevos tiempos y estilos de vida. En nuestro caso hemos podido comprobar cómo esta forma de familismo estaba presente en muchas de las tradiciones aquí señaladas y que aún permanecen arraigadas en el imaginario social colectivo, tal y como demuestran muchas de las expresiones recogidas de nuestros informantes.

Numerosos estudios sociológicos avalan la idea de la progresiva desconexión que se ha venido produciendo entre las formas de organización familiar y las formas de organización económica de la agricultura y ganadería como un signo evidente de cambio social y reestructuración del ámbito rural. Las familias de nuestra zona objeto de estudio vivían fundamentalmente de la ganadería $\mathrm{y}$ todos los miembros de la familia trabajaban en la misma actividad y lo hacían en régimen familiar. La solidaridad intergeneracional era un factor clave de la organización familiar, económica y cultural de estos pueblos. En este sentido se definían como familias "monoagrarias" donde la actividad agraria estaba regulada y gestionada familiarmente (Ruggles 2010; Sampedro 1996). Las implicaciones que esta forma de regulación familiar tiene sobre los estilos de organización social y económica son claves para entender la transformación social y cultural. De hecho estas prácticas han sido documentadas a través de las entrevistas que hemos realizado, como por ejemplo la pervivencia de un modelo patriarcal de relaciones familiares (Sampedro 1996), que ha sido la base normativa de muchas de las tradiciones que hemos documentado en este estudio. De acuerdo con Camarero y Del Pino (2014), los sistemas de familias rurales tradicionales se basaban en los modelos de familia patriarcal y troncal. En la medida en que actualmente las unidades domésticas rurales no tienen relación con la actividad familiar agraria se reduce la presencia de la "familia patriarcal" (denominada por Camarero y Del Pino (2014) "familia extensa trigeneracional"). Este tipo de organización familiar pierde su funcionalidad pero mantiene su carácter tradicional que ha sido la base-soporte de muchas tradiciones identitarias vinculadas con la economía ganadera en el ámbito rural estudiado (Aceves 1973; Gómez et al. 1999). Si bien la "familia troncal" pierde parte de su funcionalidad, sin embargo los hijos solteros que viven con sus padres siguen manteniendo el sistema que Camarero y Del Pino (2014) han denominado "familia troncal truncada", en la medida en que aunque estas unidades domésticas ya no se ocupan de la función de la productividad sí se ocupan de la reproducción de la solidaridad familiar, encargándose de dotar de nueva funcionalidad a las estructuras de convivencia generacional en el ámbito rural estudiado. La relación de intercambio de ayudas monetarias y de servicios entre generaciones en el interior de los hogares rurales no está suficientemente estudiada. Sin embargo, en el estudio realizado, a partir de las informaciones que nos han proporcionado nuestros entrevistados, hemos podido comprobar que la familia troncal en su nueva acepción sigue manteniendo parte de sus funciones de solidaridad intergeneracional convirtiéndose, por tanto, en un elemento fundamental de la cultura tradicional y de las formas de vida de esta zona rural estudiada.

En definitiva, los testimonios narrativos recogidos para este trabajo provenientes de los informantes estratégicos entrevistados apuntan a que la comunidad tradicional de estos pueblos no era la sociedad ideal de la que a veces se habla, sino más bien una comunidad que luchaba por adaptarse y sobrevivir con numerosas contradicciones a las dificultades y retos que imponía la naturaleza y la 
economía de subsistencia, siendo los testimonios sobre las tradiciones aquí recopilados un buen ejemplo de ello.

\section{Conclusiones}

El trabajo realizado ha permitido una aproximación interpretativa al estudio de la nueva ruralidad en el área delimitado de la Sierra del Guadarrama desde una perspectiva socioantropológica. Este ejercicio analítico ha permitido acercarse al fenómeno inexplorado de lo que queda de las tradiciones como efecto del proceso globalizador y modernizador en el ámbito rural castellano-leones en un entorno territorial y cultural muy concreto y definido como es la Sierra del Guadarrama segoviano en su parte más oriental.

A partir de los datos obtenidos a través de este análisis exploratorio basado en el análisis del discurso de los informantes estratégicos y de los documentos consultados podemos concluir que en esta zona objeto de estudio se ha producido una transformación estructural de las formas de producción, de los estilos de vida y de las formas de organización social, aunque manteniendo algunos rasgos tradicionales que están solo presentes en el imaginario social colectivo. Todo ello ha supuesto, en términos generales, el colofón decisivo a la quiebra del funcionamiento histórico del espacio serrano, a partir de la incidencia, sobre amplios sectores, de procesos de abandono, de despoblamiento y de prácticas de degradación. Sin embargo el análisis socioantropológico realizado ha permitido identificar algunos elementos culturales de continuidad entre el pasado y el presente. Los vestigios de las tradiciones aquí representados y relatados a través de los discursos de nuestros informantes reflejan la expresión de esa ambivalencia nostálgica entre la modernidad y la tradición, entre el recuerdo de lo que fue y de lo que no ha podido seguir siendo. Este estudio exploratorio recoge en parte la historia colectiva de un estilo de vida y forma de organización social que posibilitó formas de vida autónomas y a la vez comunitarias basadas en el aprovechamiento de los recursos naturales y ganaderos para un mercado local de subsistencia que fue sustituido por un mercado global que difícilmente podía hacer sostenible esta forma de vida.

Desde una perspectiva teórica el estudio realizado ha constado cómo las acepciones normativas de la "nueva ruralidad" y "la "hibridación cultural", responden solo en parte a la interpretación de las transformaciones culturales, económicas y productivas que se han producido en el territorio objeto de estudio. Prueba de ello es que los informantes estratégicos no se reconocen ni identifican en lo que aquí se ha denominado "nueva ruralidad". Por otra parte, estos informantes manifiestan expresamente la pérdida nostálgica de identidad cultural de estas comunidades rurales como conjunto de tradiciones pero no identifican como propia la demanda territorial de reconocimiento institucional-administrativo.

En definitiva el estudio realizado ha evidenciado que a pesar de la decadencia poblacional y económica de esta zona, ocasionada por la transformación de las estructuras económicas resultantes del proceso industrializador y globalizador, se observa una incipiente recuperación que se piensa y construye desde la sostenibilidad poblacional y económica de los nuevos pobladores rurales que mantienen algún tipo de vinculación con familiares y el pasado más inmediato de estos pueblos. Pero no puede haber recuperación de esos vestigios tradicionales que supusieron una forma de vida durante muchos años sin población y no puede haber población sin oportunidades vitales que conecten y retengan a los posibles moradores con estas zonas despobladas. Por lo tanto, el futuro de estas zonas rurales pasa por combinar la modernidad y la tradición de forma sostenible y equilibrada innovando en la forma de poner en valor la tradición como una forma de conocimiento y de valor cultural, desde el apoyo institucional a la autonomía y participación activa de los pobladores rurales de este territorio como agentes protagonistas e identificados con la "nueva ruralidad".

Agradecimientos: Este estudio ha sido subvencionado y apoyado por el Instituto de la Cultura Tradicional de la Diputación Provincial de Segovia. Se agradece muy especialmente las aportaciones y comentarios realizados por los revisores. 


\section{Referencias Citadas}

Aceves, J. 1973. Cambio Social en un Pueblo de España. Editorial Barral, Barcelona.

Aguirre Beltrán, A. 1967. Regiones de Refugio: El Desarrollo de la Comunidad y el Proceso Dominical en Mestizo América. Editorial Instituto Indigenista Americano, México, D.F.

Aitken, R. 1947. Rutas de la trashumancia en la meseta castellana. Estudios Geográficos 26 (1):185-99.

Alonso Ponga, J. 1982. Tradiciones y Costumbres en Castilla y León. Ed. Nueva Castilla, Valladolid.

Archer, M. 1988. Culture and Agency, the Place of Culture in Social Theory. University Press, Cambridge.

Arensberg, C. y T. Kimball 1965. The fringe community. En Culture and Community, pp. 135-166, Harcourt, Brace \& World, New York.

Arnhol, A. y G. Anthony 1981. Conditions of Existence in a Castilian Village: An Ethnography in Historical and National Context. Ed. University of Manitoba, Winnipeg.

Arnhold, A. 1986. Lo tradicional no es lo de siempre. En Etnología y Folklore en Castilla y León, editado por L. Díaz Viana. Junta de Castilla y León, Valladolid.

Battani, M., R. David y P. Rosemary 1997. Cultures' structures: Making meaning in the public sphere. Theory and Society 26 (6):781-812.

Biernacki, R. 2012. Reinventing Evidence for Social Inquiry: Decoding Facts and Variables. Ed. Palgrave Macmillan, New York.

Bonnell, V. y L. Hunt (eds.) 1999. Beyond the Cultural Turn. New Directions in the Study of Society and Culture. Ed. University of California Press, Berkeley.

Bretón, V., D. Comas y J. Contreras 1997. Cambio social en la agricultura familiar española. En Agricultura y Sociedad en la España Contemporánea, coordinado por C. Gómez y J. González, pp. 653-670. CIS/Ministerio de Agricultura, Pesca y Alimentación, Madrid.

Camarero, L. y J. Del Pino 2014. Cambios en las estructuras de los hogares rurales. Formas de adaptación y resiliencia. Revista Internacional de Sociología 72(2):377-401.

Caro Baroja, J. 1963. The city and the countryside: Reflections on some ancient commonplaces. En Mediterranean Countrymen: Essays in the Social Anthropology of the Mediterranean, coordinado por J. Pitt- Rivers, pp. 27-40. The Hague, Mouton.

Caro Baroja, J. 1966. La Ciudad y el Campo. Ediciones Alfaguara, Madrid.

Caro Baroja, J. 1967. Lo que Sabemos del Folklore. Ed. Gregorio del Toro, Madrid.

Catedra, M. 2008. The city and the countryside: the Virgin of Sonsoles. En Recasting Culture and Space in Iberian Contexts, coordinado por S. Roseman y S. Parkhurst, pp. 181-204. State University of New York Press, Albany.

Cazorla, J. 1994. El clientelismo de partido en España ante la opinión pública. El medio rural, la administración y las empresas. Working Paper 86/94, Institut de Ciències, Politiques i Socials, Barcelona.
Chiriboga, M. 2001. Agricultura, espacios rurales y medio ambiente en el marco de la globalización. En La Nueva Ruralidad en América Latina, tomo II, editado por E. Pérez y M. Farra Quijano, C. Castillo Rodríguez, J. Ortiz Guerrero, D. Patricia Muñoz y M. Vélez, pp. 163-187. Pontificia Universidad Javeriana, Bogotá.

De Castro, A. 1985. Del folfklore en el marco de la animación cultural. Revista de Folklore 56:61-66.

De Miguel, A. y F. Moral 1984. La Población Castellana. Ed. Ámbito, Valladolid.

Delgado, J. 1999. La nueva ruralidad en México. Investigaciones Geográficas 39:82-93.

Díez Mayoral, D. 2003. Políticas de Intervención y Dinámicas Territoriales en las Áreas del Sistema Central de Castilla y León. Tesis Doctoral. Universidad Complutense de Madrid, Madrid.

Diaz Viana, L. 1984. Rito y Tradición Oral en Castilla y León. Ed. Ámbito, Valladolid.

Diaz Viana, L. 1988. Visiones nativas y foráneas o el verdadero objeto de la etnografía: reflexiones en torno al conocimiento del patrimonio etnográfico de Castilla y León. Política y Sociedad 27:21-32.

Diaz Viana, L. 2010. Castilla y León, un territorio cuestionado: retóricas del espacio y del tiempo en la construcción de identidades. Revista de Dialectología y Tradiciones Populares 65:45-64.

Eliasoph, N. 1988. Avoiding Politics. How Americans Produce Apathy in Everyday Life. Ed. Cambridge University Press, Cambridge.

Fernández de Mosteyrín, M. y M.L. Morán 2014. Encontrar la cultura: estrategias de indagación para el análisis sociopolítico. Revista de Estudios Sociales 50:43-56.

García Canclini, N. 2004. Culturas Híbridas: Estrategias para Entrar y Salir de la Modernidad. Editorial Grijalbo, México, D.F.

García Candlini, N. y R. Roncagliolo 1989. Cultura Transnacional y Culturas Populares. Ed. Instituto para América Latina, Lima.

Geertz, C.L. 1991. La Interpretación de las Culturas. Ed. Gedisa, Barcelona.

Giddens, A. 1999. Consecuencias de la Modernidad. Alianza Editorial, Madrid.

Gilham, B. 2005. Research Interviewing. The Range of Techniques. Open University Press, Berkshire.

Gómez Echenique, S. 2002. La "Nueva Ruralidad” ¿Qué Tan Nueva? Ediciones Ltda. LOM, Santiago.

Gómez, C., J. González y R. Sancho 1999. Identidad y Profesión en la Agricultura Familiar. Ed. Centro de Investigaciones Sociológicas, Madrid.

Gómez, S. 2002. ¿Nueva ruralidad? Un aporte al debate. Estudos Sociedade e Agricultura 17:5-32.

González Herrero, M. 1998. Las Comunidades de Villa y Tierra en Segovia. Academia de Historia y Arte de San Quirce, Segovia.

Goldberg, D.T. 1994. Introduction: Multicultural Conditions. En Multiculturalism: A Critical Reader, editado por D.T. Goldberg, pp. 1-41. Basil Blackwell, Oxford. 
Günes Ayata, A. 1994. Clientelism: Premodern, modern, postmodern. En Democracy, Clientelism and Civil Society, editado por L. Roniger y A. Günes Ayata, pp. 19-28. Lynne Rienner Publishers, Boulder, Colorado.

Kay, C. 2009. Estudios rurales en América Latina en el periodo de globalización neoliberal: ¿una nueva ruralidad? Revista Mexicana de Sociología 71:607-645.

Kavanagh, W. 1986. Identidad y mundo exterior entre los habitantes de un pueblo serrano. En Etnología y Folklore en Castilla y León, coordinado por L. Díaz Viana, pp. 41-48. Ed. Junta de Castilla y León, Valladolid.

Kenny, A. 1962. A Spanish Tapestry: Town and Country in Castile. Indiana University Press, Bloomington.

Leal, J. 2000. Etnografias Portuguesas (1870-1970). Cultura Popular e Identidade Nacional. Dom Quixote, Lisboa.

Lécea y García, C. 1893. La Comunidad y Tierra de Segovia. Estudio Histórico-Legal acerca de su Origen, Extensión, Propiedades, Derechos y Estado Presente. Ed. Establecimiento Tipográfico de Ondero, Segovia.

Linck, T. 2001. El campo en la ciudad: reflexiones en torno a las ruralidades emergentes. En La Nueva Ruralidad en América Latina. Maestría en Desarrollo Rural 20 Años, volumen 1, compilado por E. Pérez y M. Farah, pp. 37-53. Pontificia Universidad Javeriana, Bogotá.

Maines, R. 2000. Charting futures for sociology: Culture and meaning. The social construction of meaning. Contemporary Sociology 29:577-584.

Manero, F. y C. Candela 1997. El Plan especial de la Sierra de Guadarrama: Marco general y estudios de apoyo. En Paisaje y Desarrollo Integral en Áreas de Montaña, editado por Varios Autores, pp. 221-236. VII Jornadas sobre el Paisaje. Ed. Ministerio de Medio Ambiente, Madrid.

Martínez Díez, G. 1983. Las Comunidades de Villa y Tierra de la Extremadura Castellana. Editora Nacional, Madrid.

Martínez Fernández, L.C. y A. Moreno Mínguez (eds.) 2015. La Provincia de Segovia. Interpretación del Espacio y Definición del Modelo Territorial. Diputación de Segovia, Segovia.

Molina de La Torre, I. y J.M. Delgado Urrecho 2014. Una estructura territorial contrastada. En La Provincia de Segovia. Interpretación del Espacio y Definición del Modelo Territorial, pp. 353-414, editado por L.C. Martínez Fernández y A. Moreno Mínguez. Diputación Provincial de Segovia, Segovia.

Montes Pérez, C. 2001. Cultura ganadera de la comarca de Sayago. En Estudios de Etnología en Castilla y León 1992-1999, editado por Varios Autores. Ed. Junta de Castilla y León, Valladolid.

Montesino González, A. 1982. Las Marzas: Rituales de identidad y Sociabilidad Masculinas. Editorial Límite, Santander.

Nadal, J. 1975. El Fracaso de la Revolución Industrial en España. Ed. Ariel, Barcelona.

Narotzky, S. 1991. La Antropología de los Pueblos de España. Icaria, Barcelona.

Nogué, J. 2005. El fenómeno neorural. Agricultura y Sociedad 47 (1988):145-175.

Pereiro, X. y S. Prado 2005. Turismo e oferta gastronómica na comarca de Ulloa (Galiza): Análise de uma experiência de desenvolvimento local. PASOS. Revista de Turismo y Patrimonio Cultural 3 (1):109-123.

Plaza Gutiérrez, J., M. Martín Jiménez, I. Hortelano, L. Mínguez y R. Fernández Álvarez 2010. Urbanización de la montaña. Intensidad y alcance del proceso; formas y paisajes inducidos en algunas comarcas del Sistema Central. X Coloquio y Jornadas de Campo de Geografía Urbana. Espacios y Paisajes Urbanos. Reflexionar sobre su Presente para Proyectar su Futuro, editado por Varios Autores, pp. 289-303. Ed. Asociación de Geógrafos Españoles. Universidad de Oviedo. Universidad de Cantabria y Universidad del País Vasco, Oviedo.

Pitt-Rivers, J. 1961. The People of the Sierra. Ed. Phoenix Books y The University of Chicago Press, Chicago.

Pitt-Rivers, J. 1984. La identidad local a través de la fiesta. Revista de Occidente 38-39:17-35.

Polletta, F. 2008. Story telling in politics. Contexts 7 (4):26-31.

Prado S. 2007. Novas Minorías nas Institucións Educativas. Sotelo Blanco, Santiago de Compostela.

Ribas Palom, A. y D. Saurí Pujol 2006. De la geografía de los riesgos a las geografías de la vulnerabilidad. En Las Otras Geografías, editado por J. Nogué y J. Romero, pp. 285-300. Tirant lo Blanch, Valencia.

Roberts, B. 1978. Cities of Peasants: The Political Economy of Urbanization in the Third World. Ed. Edward Arnold, Londres.

Rodríguez Campos, X. 2008. Negotiating urban post-modern identities in Galicia: The recovery of rural dwellings. International Journal of Iberian Studies 21 (2):151-170.

Roseman, S., S. Prado y X. Pereiro 2011. Antropología y nuevas ruralidades. Introducción teórica. Actas del XII Congreso de Antropología de la FAAEE, pp. 2163-2172. Ed. Asociación de Antropología de Castilla y León, Valladolid.

Ruggles, S. 2010. Stem families and joint families in comparative historical perspective. Population and Development Review 36:563-577.

Ruiz, N y J. Delgado 2008. Territorio y nuevas ruralidades: un recorrido teórico sobre las transformaciones de la relación campo-ciudad. Revista Eure XXXIV (102):77-95.

Sampedro, R. 1996. Género y Ruralidad: las Mujeres ante el Reto de la Desagrarización. Instituto de la Mujer, Madrid.

Schejtman, A. 1999. Urban dimensions of rural development. CEPAL Review 67:15-33.

Sewell, J. 1992. A theory of structure: duality, agency and transformation. American Journal of Sociology 91:1-29.

Somers, M. y F. Block 2005. From poverty to perversity: Ideas, markets and institutions over 200 years of welfare debates. American Sociological Review 70 (2):260-287.

Sorokin, P., C. Zimmerman y C. Galpin 1981 [1930]. A systematic source book in rural sociology, The University of Minnesota Press, Mineapolis. En Introdução Critica a Sociología Rural, editado por J. De Souza Martins. Editorial Hucitec, São Paulo.

Swidler, A. 1986. Culture in action: Symbols and strategies. American Sociological Review 51:273-286.

Töennies, F. 1973. Comunidade e sociedade como entidades típicoideais. En Comunidade e Sociedade. Leitura sobre Problemas 
Conceituais, Metodológicos y de Aplicação, editado por F. Fernándes. Editora da Universidade de São Paulo, São Paulo.

Troitiño Vinuesa, M.A. 1990. El sistema central. En Geografía de Castilla y León, editado por A. Cabo Alonso y F. Manero Miguel, pp. 76-139. Ed. Ámbito, Valladolid.

Velasco Maillo, H. 1988. El papel del Folklore y de la cultura popular en la construcción del sentido de diferencia. En Democracia y Diferencia. Cultura, Poder y Representación en los Estados Unidos y en España, editado por D. Greenwood y C. Greenhouse, pp. 333-356. Ed. UNED.
Velasco Maillo, H. 2001. Caracterizaciones etnográficas y señas de identidad. Análisis antropológicos sobre Castilla y León. En Estudios de Etnología en Castilla y León 1992-1999, editado por Varios Autores, pp. 31-52. Ed. Junta de Castilla y León, Valladolid.

Velasco Maillo, H. 2004. La sociedad como mercado. Roles y grupos en mercados y ferias de ganado en Castilla y León. Revista de Estudios 51:193-216.

Vallés, M.S. 2002. Entrevistas Cualitativas. Cuadernos Metodológicos 32. CIS, Madrid.

Wolf, E. 1975. Los Campesinos. Labor, Barcelona.

\section{Notas}

1 Sin ánimo de hacer aquí un ejercicio de erudición sobre las antiguas demarcaciones territoriales de las Comunidades de Villa y Tierra (o de Ciudad y Tierra, en el caso único de Segovia) segovianas, se anima a la consulta, para los interesados en este tema de evidente entronque con algunos de los aspectos más destacados de la tradición cultural, de los estudios llevados a cabo por González Herrero (1998) y Martínez Díez (1983). Para el caso particular de la Comunidad de Ciudad y Tierra de Segovia es digna de mención la monografía escrita por Lécea (1893), en la que se detallan cuestiones relativas a su origen, extensión y propiedades históricas.

2 Un espacio que, precisamente, por los valores culturales que atesora fue incluido plenamente dentro del Plan Especial de la Sierra de Guadarrama en Segovia (Alonso 1997; Manero y Candela 1997). Un Plan que, aunque no fuera llevado a la práctica, a pesar de lo avanzado que estaba su marco general y los estudios de apoyo que se realizaron, puede considerarse como uno de los primeros antecedentes, junto con el Estudio previo para la declaración de la Sierra de Guadarrama como Espacio Natural Protegido de 1991, del "Parque Natural Sierra Norte de Guadarrama", declarado por la Junta de Castilla y León en 2010.

3 Para más información sobre la "Entrevista" como técnica de investigación social ver Gilham (2005) y Vallés (2002).

4 Chiriboga (2001) utiliza el concepto de "desanclaje" de Giddens (1999) para referirse a la transformación de las actividades sociales en el ámbito rural en los procesos de interacción como consecuencia de los procesos de globalización (aumento de las distancias espacio-temporales) y sus implicaciones transformadoras en el territorio y en las relaciones sociales. 

ANDROS IMPRESORES

www.androsimpresores.cl 